\title{
Main technical authors
}

User's guide

Part I:

System design

SL corpus design

SL corpus collection

SL corpus representation

Part II:

SL lexica

Language models

Physical characterisation and description

Part III:

Assessment methodologies and experimental design

Assessment of recognition systems

Assessment of speaker verification systems

Assessment of synthesis systems

Assessment of interactive systems
Roger Moore

Khalid Choukri

Els den Os

Christoph Draxler

Els den Os

Dafydd Gibbon

Hermann Ney

Lars Knohl \& Volker Kraft

Peter Howell

David van Leeuwen \& H. Steeneken

Frédéric Bimbot \&

Gérard Chollet

Renée van Bezooijen \&

Vincent van Heuven

Norman Fraser

Part IV:

Contributions from named Projects Main new contributions

Christoph Draxler, John Esling, and revisions

John Wells 
